\title{
Failure Recovery with Shared Autonomy
}

\author{
Bharath Sankaran, Benjamin Pitzer and Sarah Osentoski
}

\begin{abstract}
Building robots capable of long term autonomy has been a long standing goal of robotics research. Such systems must be capable of performing certain tasks with a high degree of robustness and repeatability. In the context of personal robotics, these tasks could range anywhere from retrieving items from a refrigerator, loading a dishwasher, to setting up a dinner table. Given the complexity of tasks there are a multitude of failure scenarios that the robot can encounter, irrespective of whether the environment is static or dynamic. For a robot to be successful in such situations, it would need to know how to recover from failures or when to ask a human for help.

This paper, presents a novel shared autonomy behavioral executive to addresses these issues. We demonstrate how this executive combines generalized logic based recovery and human intervention to achieve continuous failure free operation. We tested the systems over 250 trials of two different use case experiments. Our current algorithm drastically reduced human intervention from $26 \%$ to $4 \%$ on the first experiment and $46 \%$ to $9 \%$ on the second experiment. This system provides a new dimension to robot autonomy, where robots can exhibit long term failure free operation with minimal human supervision. We also discuss how the system can be generalized.
\end{abstract}

\section{INTRODUCTION}

For robots to be successful in domains outside industrial applications, a fundamental requirement that needs to be addressed is long term autonomy. Ideally one would like to restrict human supervision to tasks such as initiation of a desired activity or intervention during failure. Since personal robots enagage in complex interactions with their environment, achieving this level of robustness becomes a challenging task. As Meeussen et al [1] have noted, in the event of a failure the robot should be capable of asking for help, but the question remains as to how and when this should be done. In this paper, we examine some of these questions and provide a framework to address them.

Robot autonomy is an extremely difficult task even in highly constrained environments. In the personal robotics domain, robotic assistants are expected to perform roles similar to that of a maid or a busboy: clearing a table, getting drinks from a refrigerator etc. In the current state-of-the-art high level tasks like these are accomplished through executives which execute high level robot actions to accomplish these tasks. Such a task level executive architecture has been demonstrated by Bohren et al in the SMACH system [2]. Such systems are useful as they are specified via high level tasks which are analagous to human instruction. This form

B. Sankaran is with the GRASP Laboratory, University of Pennsylvania, Philadelphia, PA 19104, USA., bharath@ seas . upenn. edu

B. Pitzer and S. Osentoski are with Robert Bosch LLC at the Research and Technology Center North America, Palo Alto, CA 94304, USA., \{Benjamin.Pitzer, Sarah.Osentoski\}@us.bosch.com

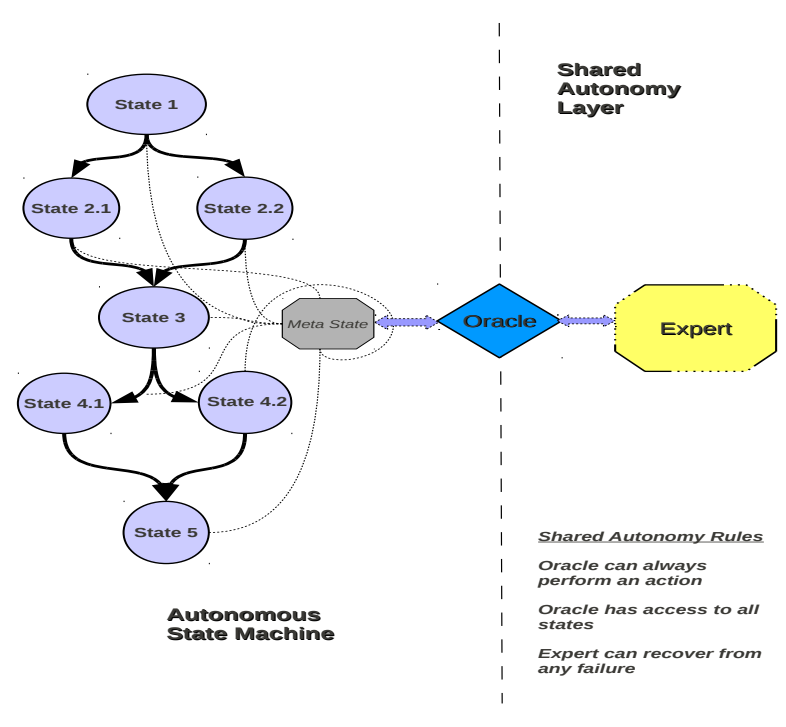

Fig. 1. Shared Autonomy Topological Representation: This representation shows how the oracle forms a meta state which enables transitions between all states in the state machine. It also behaves as the interface between the human expert and the state machine.

of a system enables rapid prototyping for application development in comparison to imperative scripting approaches or model based task-planners. Task executives primarily act as a procedure definition system for task planners, which make them extremely flexible and reusable. Though general enough to incorporate any robot action to accomplish a task, such systems are highly prone to failure. Since these executives operate on high level actions, any system built to recover from the failure of these high level actions needs do so without the knowledge of the underlying low level mechanisms. Also when scripting these systems to recover from failure, it is not possible to account for every failure case during the design phase. In these scenarios identifying the source of failure and troubleshooting the specific subsystem is not plausible due to the unknown complexity of the underlying system.

In this paper, we present an architecture which performs logic based recovery actions on such task executives. Since these task executives are defined as state machines, the recovery architecture operates on the states of the state machine to enable efficient failure recovery. The recovery architecture incorporates an expert interface to enable human intervention in the case of unrecoverable failures. The system also adaptively learns an online failure rate for each action in the state machine to determine the level of human 
intervention required in the event of a failure. Though failure detection is a crucial aspect for failure recovery, it is an entirely separate research topic in itself. For the sake of this work we assume that the system has the inherent ability to detect failure. The novel contributions of this work are :

1) A generalized logic based recovery algorithm where the user does not need to identify every possible failure case.

2) A novel method for combining expert interfaces to assist in failure recovery through shared autonomy.

In the following sections, we describe the related work to this paper, followed by a System Description and Overview of task executives and shared autonomy. Then we elaborate on the Behavioral Executive followed by the Expert Interface. In the final two sections we present our Experimental Results and Conclusions.

\section{RELATED WORK}

Long term autonomy has been addressed by many researchers in their system specific capacities. Robots which aim for long term autonomy have been demonstrated in the numerous cases, for example where robots have been used tour guides. To address failure recovery in these robotic systems, varying levels of human intervention are directed towards altering the environment of the robot to ensure successful operation.

RHINO [3], the first robotic tour guide, subsequently MINERVA [4], Mobots [5], Robox [6] and Jinny [7] navigate around museum environments with varying degrees of success. Since these robots operated in environments which were highly dynamic, their success depended on efficient localization. Hence occasional modifications had to be made to their map of the environment to ensure their localization was accurate. This form of human intervention involves directly altering the environment to ensure that the robots can function in a robust fashion. Though this technique is effective for localization tasks, in most robot autonomy tasks modifying the environment can have unintended consequences, as some modes of the operation of the robot may depend on its perception of the environment.

In the space exploration industry, where failure free operation is absolutely critical to mission success [8], systems tend to demonstrate a high level of robustness by either combining autonomy and human input or relying entirely on human assisted operation. Though the problem of generalized task level failure recovery has not been explicitly addressed, task specific recovery has been addressed by previous work. There have been two areas of research in this field. One where task specific recovery behaviors are scripted into the system, like the ability to handle navigation failures in the Urban Car Challenge [9]. The other where some form of human input has been injected into a system to achieve a high level of robustness in human environments. Researchers at Bosch [10] and Willow Garage [11] have also argued for human intervention in robotic systems for long term autonomy, but the level of human intervention required still seems to be an open research question. We try to address some of these issues in this paper.

\section{System Description ANd OVERVIEW}

In our system, the front end consists of the human interaction layer which includes a shared autonomy interface, a state machine viewer and sensor data visualizer. The user interacts with the robot through this layer. The back end of the system which consists of the robot which has two layers of abstraction. A task executive layer and a layer which is a collection of the low level subsystems. The task executive takes each high level action and executes it by calling the appropriate low level subsystem. The low level subsystems are divided into three main modules: planning, perception and control. As the task executive calls each of these modules, the modules execute the appropriate action corresponding to each task. For example when the executive executes a pick object action, the action layer would call the grasp planner and the arm controller modules to successfully execute the action. The generalized architecture for our system is depicted in Fig 2.

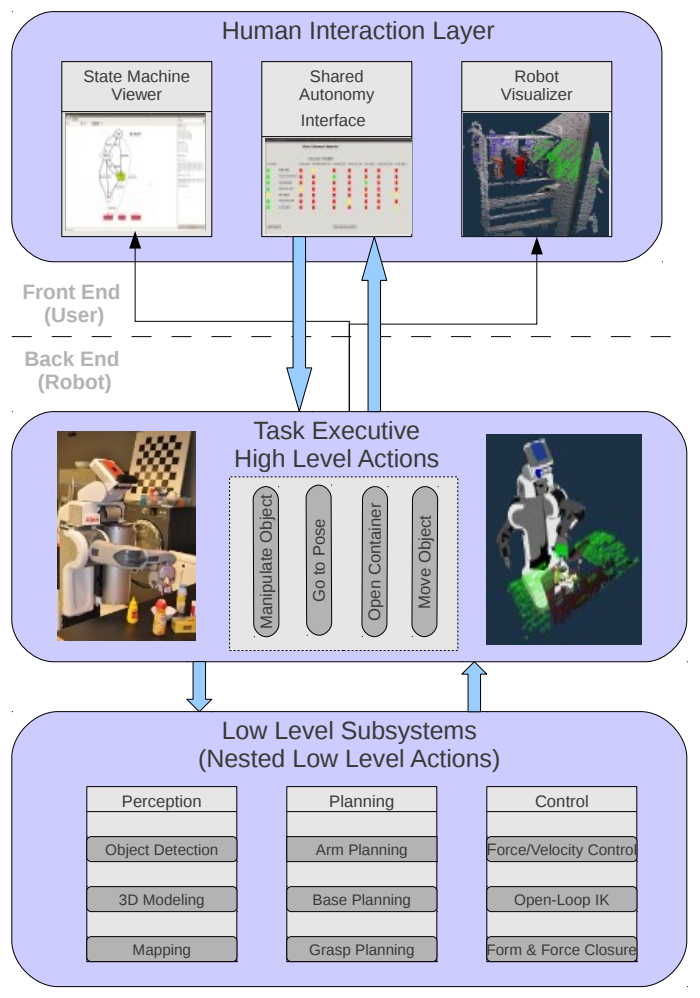

Fig. 2. System Architecture: The front end of the system comprises of the human interaction layer. The back end, which is present in the robot, consists of the task executive which translates high level actions in to low level commands which can be executed by the planning, perception and control modules of the robot.

High level robot tasks can be broadly classified as either some form of information acquisition, information analysis, decision making or plan execution. Since these high level tasks are used in a task executive, our shared autonomy interface directly interacts and replaces these tasks in the 
event of an expert query. We define shared autonomy as a partial or full replacement of an action that the robot has to execute to accomplish a specific task.

In this paper we use this definition of shared autonomy to define levels of human intervention for failure recovery while keeping the original system fully autonomous. During an expert query, the user has the ability to intervene and supersede any stage of the robot's task execution pipeline. Though there have been attempts to preempt failure through planning [12], our system addresses situations which require recovery since all failures cannot be preempted.

\section{BEHAVIORAL EXECUTIVE}

Most state machines that are designed for robot applications are non-ergodic in nature, i.e every state cannot be reached from every other state in the state machine. The nonergodicity of these state machines makes task level recovery impossible, as the state machines cannot revisit states that it had occupied before the current failure state. This is demonstrated in the example state chart [13] in Fig 3(a). For instance if the action in state FOB fails, the state machine fails. Designing recovery behaviors in this state machine is trivial, given its relative simplicity. The state machine could be redesigned to revisit a select set of states on the event of a failure. Though trivial given the number of states in the state machine, this approach cannot be generalized as it is tough to reason about relations between states in large state machines. This tends to be the case in state machines designed for most robot applications. In general, robot state machines to perform a certain task can be extremely complex. Unless explicitly defined by the designer of the state machine, crafting recovery behaviors to account for all possible failures is non trivial and generally intractable. To address this specific issue we introduce a generalized failure recovery mechanism, through the introduction of a meta state called the oracle.

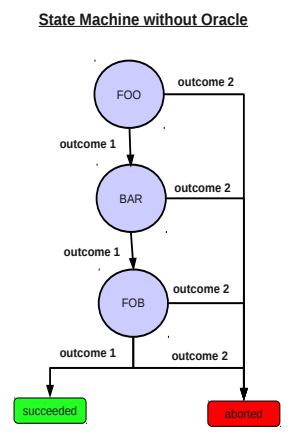

(a)

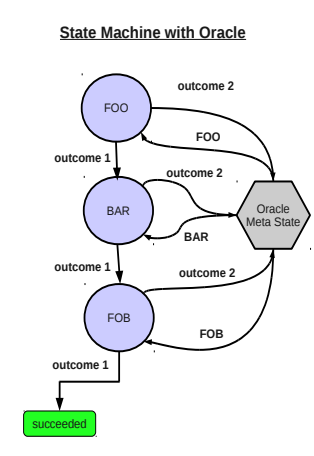

(b)
Fig. 3. Ergodicity with the Oracle: The State machine without an oracle displays non ergodic behavior. The State machine with the oracle demonstrates how the addition of the oracle makes the state machine ergodic

\section{A. Oracle State}

The oracle is a meta state which can help the state machine transition from any failed state to any other state in the state machine. When the state machine is represented as a graph, then the introduction of the oracle makes all nodes in the graph accessible as it shares an edge with every node in the graph. This is illustrated in Fig 3(b). If an action fails, it results in the failure of the state executing the action. In this case, the oracle executes a set of logic based recovery behaviors before querying the expert. These logic based recovery behaviors either execute a set of preconditions for the current state to succeed or transition the state machine to a previous state without any preconditions. States without preconditions are Markovian and hence transitioning to them does not violate any consistencies in the state machine. The oracle also adaptively learns a limited memory failure rate for each state. Since this memory is a tunable parameter, the frequency of expert intervention can be set by the designer of the state machine. This enables the oracle to classify the states a LOW_FAILURE, MODERATE_FAILURE and HIGH_FAILURE states. In the event of encountering a high failure state, the oracle has the ability to query an expert for help. The advantage of the oracle state is that reduces the design complexity of the state machine. In a typical state machine with $\mathrm{n}$ states, the number possible transitions would be $n^{2}$. If the state machine were to be designed to account for all possible failure recovery mechanisms, the designer of the state machine would have to reason about $O\left(n^{2}\right)$ transitions. The complexity of such a system would increase quadratically with the increase in the number of states. Though solvable in polynomial time, this process would be highly memory intensive. With the addition of the oracle we restrict the reasoning to a fixed number of states and also enable expert intervention. The oracle's shared autonomy recovery algorithm is illustrated in Algorithm 1.

\section{B. Preconditions}

To successfully execute most actions present in a state, there needs to be a set of preconditions that need to be satisfied which are crucial to the success of that task. Defining the preconditions, for a state can be determined by the designer of the state machine based on the actions being carried out in the state. For example, if you consider the action of grasping an object, the preconditions of this action would be :

a) An object has been detected

b) A collision map for the environment is available

c) The gripper is empty.

Since these preconditions are task specific and determining them is strictly based on the design of the system, the designer can specify these preconditions in the state machine design in our current system. During the normal execution of tasks the preconditions wouldn't not be invoked as they would have been satisfied by previous actions carried out by the state machine. These preconditions are invoked only when a particular action fails. The precondition handling is controlled by the oracle 


\section{Recovery Algorithm}

The recovery algorithm illustrated in Algorithm 1, details the various stages of recovery the system undergoes in the event of a failure. When an action in a given state fails on execution, the state machine enters the failure recovery mode. In this mode the algorithm first checks if the current state was the most recent failed state, this helps ascertain continuous failure of a given state. Once determined if the current failed state does not have any preconditions the most recent visited state is set to the current state. This helps the oracle revisit the current state in the case that the current state is not a continuous failure state.

Once the preconditions have been determined, the oracle satisfies these preconditions if any (for the low and moderate failure cases), and executes a failure mode specific - recovery strategy. The failure rates of each mode are initialized during the design phase. This heuristic helps tune the level of allowable expert intervention for each state. As the state machine runs the failure rates for each state is learned online, where influence of the prior is diminished by means of a finite memory. This approach helps the oracle easily adapt to the behavior of the system in reaction to its environment. In the case of a high failure rate state, oracle directly queries the expert on failure. In the case of low and moderate failure modes, if the failed state is not deemed as a continuous failure state, the oracle revisits the current state. In contrast, if the failed state is classified as a continuous failure state then: in the case of a low failure rate state the state machine transitions to the last known successful state preceding the failed state, in the case of a moderate failure state the algorithm queries the expert.

\section{EXPERT INTERFACE}

When the oracle queries the expert on the event of an action failure, the expert through an interface can transition the state machine to any state in the state machine for recovery. The expert also has the ability to supersede failure states through user defined shared autonomy interfaces and subsequently transition the state machine into a new state. These shared autonomy interfaces can be used to replace specific actions in the states of the state machine. This solution is particularly useful when the robot encounters situations which are unresolvable autonomously. For instance if a perception action fails to segment an object of interest, the expert can intervene with a user assisted segmentation interface. This will allow the expert to supersede the state performing the perception action and continue with the execution of the state machine. The assumption in this approach is that ultimately the expert can resolve all failures. The two shared autonomy interfaces that we provide and use in our frame work are a perceptual shared autonomy interface and a manipulation shared autonomy interface.

\section{A. Shared Autonomy}

In the perceptual shared autonomy interface, we replace the robot's object detection module with a human assisted interface. The human assisted perception interface lets the

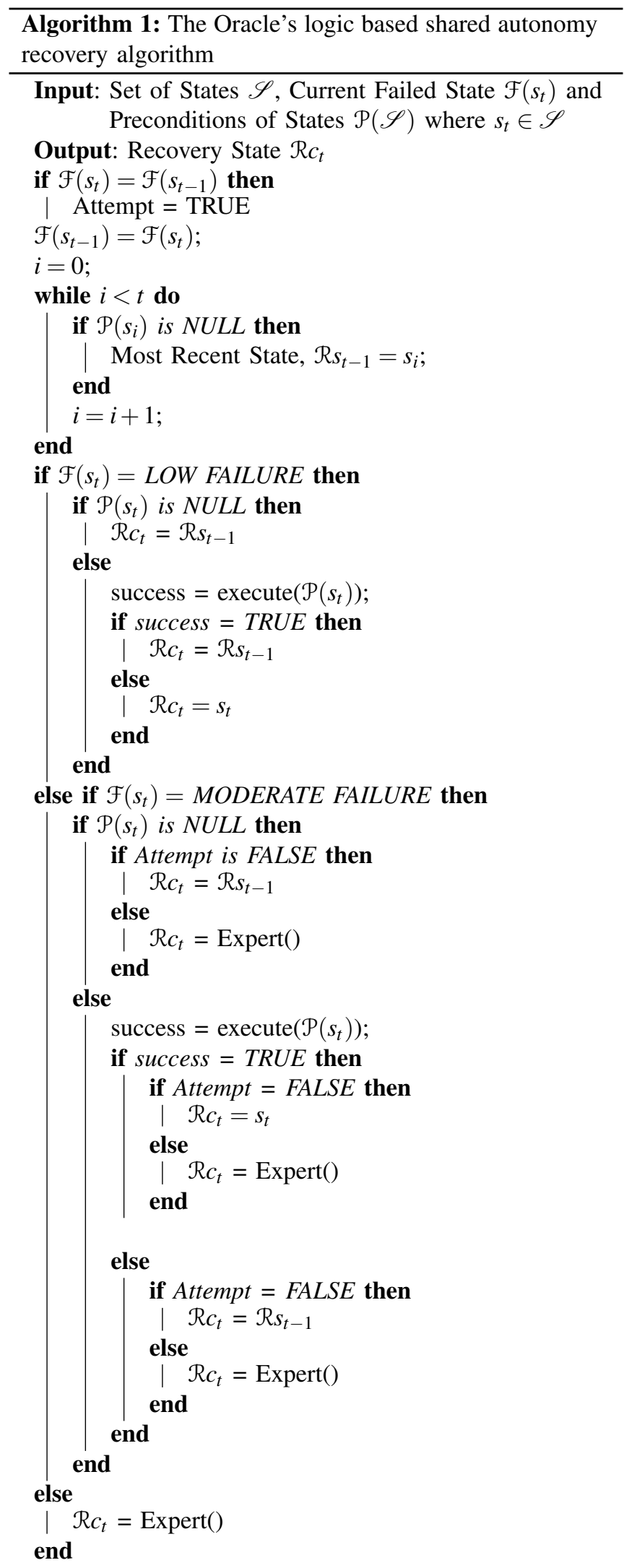

return $\mathcal{R} c_{t}$; 
human select a region of interest and the lets the system estimate the object based on the grabcut algorithm [14]. Similarly, the manipulation shared autonomy interface consists of a 6 degree of freedom teleoperation module which enables the expert to supersede the planning and control phase during manipulation failures. During the execution of the behavioral executive, in the event of an expert query, the expert can use these shared autonomy interfaces to replace any specific action performed by the robot and then transition the robot state machine to the state that follows the successful completion of the current state. This method allows the seamless integration of expert assistance into a shared autonomy environment without having to stop continuous operation.

\section{Shared Autonomy Interfaces}
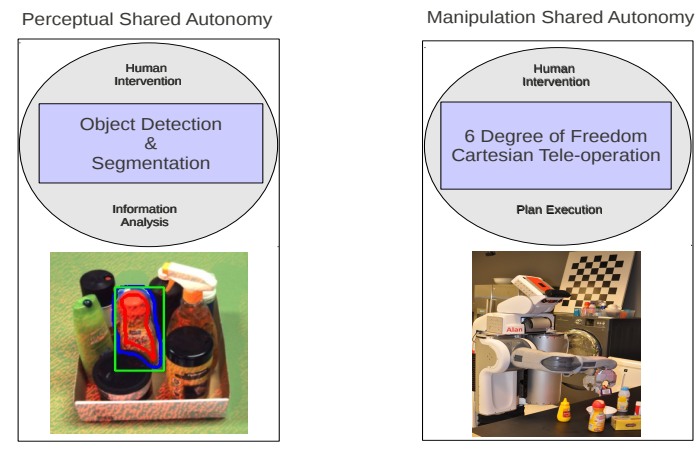

Fig. 4. The following diagram shows two shared autonomy interfaces. 1) The Perceptual Shared Autonomy interface is used to replace object detection with human assisted object detection and segmentation. 2) The Manipulation Shared Autonomy interface is used to replace planning and control with a 6 degree of freedom teleoperation device.

\section{EXPERIMENTS}

To demonstrate the efficiency of our approach, we have built our system on top of the existing SMACH [2] architecture present in ROS [15]. SMACH is a task executive where tasks can be specified in the form of a state machine. The state machine is defined in terms of states, where these states consist of actions that need to be executed. Transitions are enabled by mapping the outcome of these states to the other states in the state machine.

\section{A. SMACH Failures}

In SMACH, tasks relating to the accomplishment of a goal are specified as states in a state machine. Based on the outcome of the task execution, the state machine transitions from one state to another. In the underlying definition of a SMACH state machine, every outcome of the state in the state machine is mapped to a transition. In the event of an unsuccessful outcome, the state machine either transitions to some user specified state or it aborts. The aborted state machine would technically result in a failed attempt at the task. Our experiments were performed on state machines designed for the PR2 [16] robot built by willow garage. We tested the system on two separate use cases. The first use case involves a simple pick and place operation and in the second use case, the robot is commanded to retrieve a drink from the refrigerator. The operation of both use cases are illustrated in the accompanying video.

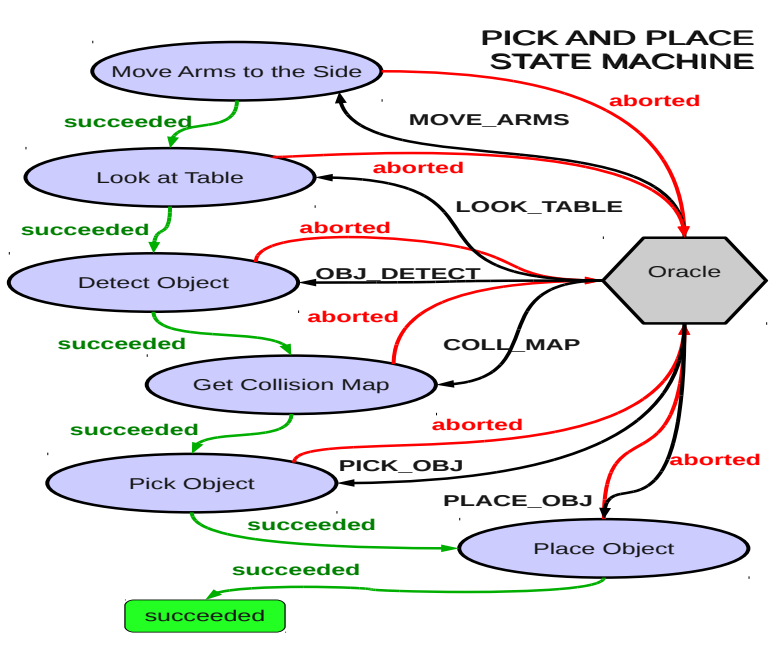

Fig. 5. State machine for the pick and place operation

\section{B. Use Cases}

1) Use Case 1: Pick and Place Operations: In our first use case, we evaluate the robot's long term performance on a simple pick and place operation. Here the robot is tasked with detection an object on a tabletop and moving it to a new location. The order of actions performed by the robot include, moving the arms out of the line of sight $\rightarrow$ pointing the head of the robot $\rightarrow$ detecting tabletop $\rightarrow$ estimating collision map $\rightarrow$ picking object $\rightarrow$ placing object. As earlier noted these are high level task specifications outlined in the task executive and they do not detail low level behavior. The low level behavior embedded into these tasks are not visible to the task executive. The state machine of this system with the oracle is illustrated in Fig 5.

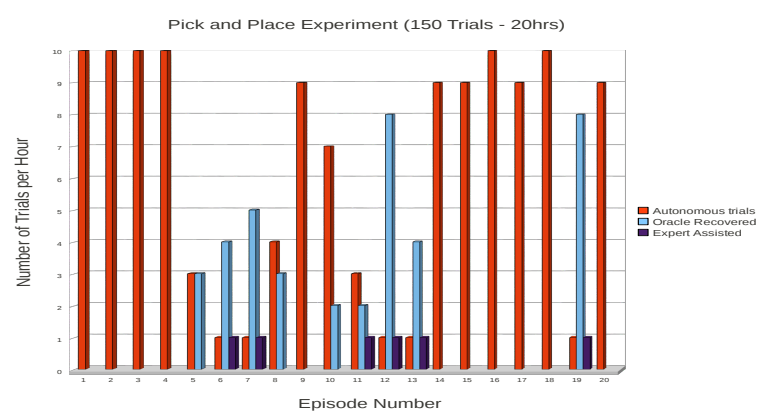

Fig. 6. Time series graph showing frequency of oracle recoveries and expert assists in 150 trials of autonomous operation over 20 hours. 
We performed this test for 150 cycles over a period of 20 hours. The most common modes of failure in this test were either perception failures, where the perception system failed to detect the object or planning failures where the arm planner could not find a valid collision free path to the desired location. In the first type of failure, a simple step back strategy would have resulted in the state machine reverting to the previous high level state where it estimates the collision map. This step back procedure would have repeated on each trial of the failed state, rendering the state machine idle without making any progress towards the completion of the task. Since the perception failure was the caused from either an arm of the robot blocking the view of the sensor or the failure of the segmentation algorithm, the recovery algorithm was able to effectively account of these failures and recover from them.

In the case of the sensor being blocked, the state machine would look to satisfy the preconditions of the object recognition state which specified that both of arms of the robot should stay clear of the line of sight of the sensor. This would cause the state machine to execute these preconditions and then proceed towards successful task recovery. In the case where the segmentation algorithm failed, the oracle would attempt multiple recovery procedures, on each continuous failure recovery attempt the failure rate of the current state would increase. This would eventually lead to the oracle querying the expert. The expert with the assistance of the shared autonomy object detection interface recovers from the failed state and then transitions the robot to the next state, thereby ensuring successful task completion.

2) Use Case 2: Drink Retrieval: In our second set of experiments we tested the algorithm on a drink retrieval state machine. Here the robot is tasked with retrieving a drink from a refrigerator. The level of complexity involved in the drink retrieval experiment is much higher than that of the pick and place operation. Thus the robot state machine includes far greater number of states and is more prone to failure. The order of actions performed by the robot include, raising the torso $\rightarrow$ opening the fridge $\rightarrow$ moving into the fridge $\rightarrow$ detecting the drink $\rightarrow$ picking the drink $\rightarrow$ moving out of the refrigerator $\rightarrow$ closing the door $\rightarrow$ moving to home position $\rightarrow$ handing the drink. The state machine of this system with the oracle is illustrated in Fig 7.

We performed this test for 100 cycles over a period of 16 hours. The most common failure resulted from the failure of the perception algorithm where the robot could not detect the drink. Due to the complexity of the system, the perception failure had resulted from variety of different causes during the various trials. Some failures were the outcomes of other undetected failures during the execution of the pipeline. For instance, in cases where the perception system failed because the refrigerator door was not completely open during the run, the system checks whether the preconditions of the perception state have been satisfied. Had this recovery been crafted as a simple step back procedure the state machine would have cycled between the positioning and perception

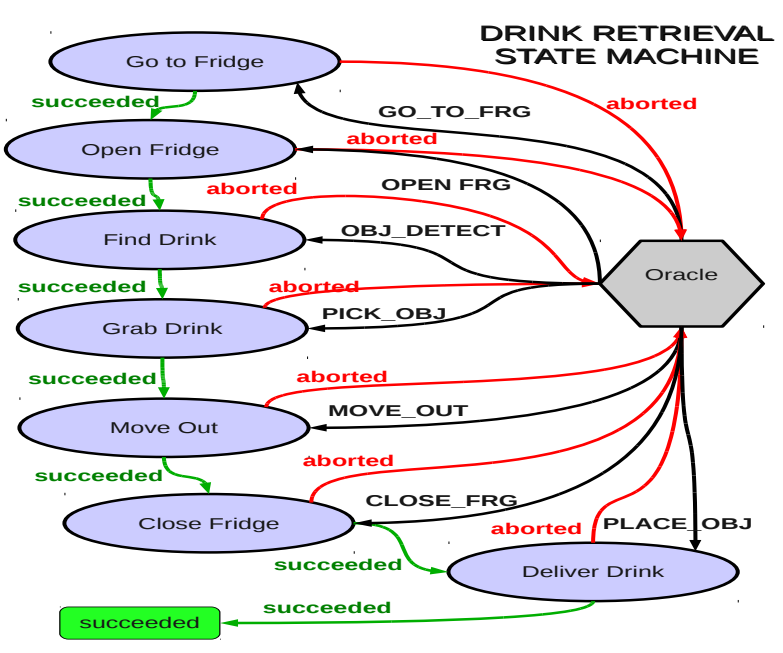

Fig. 7. State machine for the Drink Retrieval Operation

states in the state machine. In our case, the system in its attempt to satisfy the preconditions reopens the door of the refrigerator and revisits the perception state, there by eventually succeeding. In simple perception failures which result due to a failure of the detection algorithm the system re-executes the detection pipeline and eventually succeeds.

In other cases of failure which include planning failure, as the failure rate of the state increases it becomes a high failure state which introduces expert intervention through a tele-operation shared autonomy interface. Once the expert supersedes the planning and control phase with the teleoperation module he/she can transition the state machine to the next subsequent state.

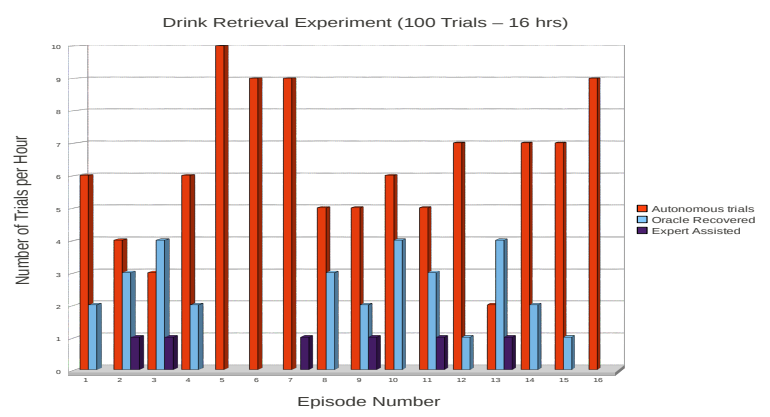

Fig. 8. Time series graph showing frequency of oracle recoveries and expert assists in 100 trials of autonomous operation over 16 hours.

As it can be noted from Table I, In both the experimental trials, the introduction of an oracle drastically reduces the level of human supervision required in such systems. In the absence of the oracle, most recovery trials would have resulted in human intervention. Given these statistics we can note that human intervention for the pick and place experiment was reduced from $26 \%$ to $4 \%$ and in the drink retrieval experiment, human intervention was reduced from 
TABLE I

SHARED AUTONOMY STATISTICS

\begin{tabular}{|c|c|c|c|}
\hline Experiment & Runs & Oracle Recoveries & Expert Interventions \\
\hline Pick and Place & 150 & 39 & 6 \\
\hline Drink Serving & 100 & 46 & 9 \\
\hline
\end{tabular}

46\% to $9 \%$. On an average we get a $\mathbf{5 0 0 \%}$ improvement in performance.

\section{Discussion \& FUTURE WORK}

In order to attain the goal of long term autonomy, robotic systems need to be made both reliable and robust. However this cannot be exclusively achieved simply through task-level robustness since task-level robustness is only one component of any robotic system framework. There needs to be a considerable emphasis on system-level robustness too. An important design feature for robots systems would include ability to recover from failure. Simply because these systems cannot be designed to preempt every possible failure case.

In this paper, we have outlined a generalized task based recovery architecture which can be readily applied to any robotic system. From our experiences in continuous operations we argue that shared autonomy is an imperative component to achieve reliable long term operation in robotic systems. Sparsely supervised systems with effective failure recovery strategies can help achieve otherwise unattainable goals in robotic applications.

In our work we also present a simple and novel method where the number of transitions an expert needs to reason about reduces from quadratic to a constant number of states. This approach vasity reduces the complexity of the system. Our system has shown significant improvements over using traditional state machines and it can be used to efficiently augment any traditional state machine. In the future we also intend to model these systems to constrain the search space of possible recovery behaviors. All the software used in this paper is readily available for download on www.ros.org.

\section{A. Unrecoverable Failures}

Though the oracle based recovery can account for task failures, the system however does not account for non software related failures. These would come under the general umbrella of unrecoverable failures, as they cannot be addressed from the software side. This would include kernel panics, hardware failures, electronic failures and battery problems. The only realistic recovery possible in such cases is to store in the state of the system externally and debug the system. Then the robot can be restarted from its externally stored state.

\section{ACKNOWLEDGEMENTS}

We would like to like to thanks Dejan Pangercic of Technische Universität München and Christian Bersch of Bosch RTC for their insightful comments and help in setting up the use case experiments. We would also like to Chris
Mansley of Rutgers University and Dr. Philip Roan of Bosch RTC for their comments and critiques. Last but not the least we like to to thank Dr. Jan Becker the head of the robotics group at Bosch RTC, for his support and guidance.

\section{REFERENCES}

[1] W. Meeussen, E. Marder-Eppstein, K. Watts, and B. Gerkey, "Long term autonomy in office environments," in ALONE Workshop,In Proceedings of Robotics: Science and Systems (RSS'11), Los Angeles, USA, 2011.

[2] J.Bohren, R. B. Rusu, E. Jones, E. Marder-Eppstein, C. Pantofaru, M. Wise, L. Mösenlechner, W. Meeussen, and S. Holzer, "Towards autonomous robotic butlers: Lessons learned with the pr2," in International Conference on Robotics and Automation (ICRA'11), Shanghai, China, May 2011.

[3] S. Thrun, A. Bücken, W. Burgard, D. Fox, T. Fröhlinghaus, D. Henning, T. Hofmann, M. Krell, and T. Schmidt, "Map learning and high-speed navigation in RHINO," in AI-based Mobile Robots: Case Studies of Successful Robot Systems, D. Kortenkamp, R. Bonasso, and R. Murphy, Eds. MIT Press, 1998.

[4] S. Thrun, M. Bennewitz, W. Burgard, A. Cremers, F. Dellaert, D. Fox, D. Hähnel, C. Rosenberg, N. Roy, J. Schulte, and D. Schulz, "MINERVA: A second generation mobile tour-guide robot," in Proceedings of the IEEE International Conference on Robotics and Automation (ICRA), 1999.

[5] I. R. Nourbakhsh, C. Kunz, and T. Willeke, "The mobot museum robot installations: a five year experiment," Proceedings 2003 IEEERSJ International Conference on Intelligent Robots and Systems IROS 2003, vol. 4, no. October, pp. 3636-3641, 2003.

[6] R. Siegwart, "Robox at expo.02: A large-scale installation of personal robots," Robotics and Autonomous Systems, vol. 42, no. 3-4, pp. 203222, 2003.

[7] G. Kim, W. Chung, K. rock Kim, and M. Kim, "The autonomous tourguide robot jinny," in In Proceedings of the IEEE/RSJ International Conference on Intelligent Robots and Systems (IROS, 2004, pp. 34503455.

[8] M. Maurette, "Mars rover autonomous navigation," Auton. Robots, vol. 14, pp. 199-208, March 2003.

[9] C. Baker, D. I. Ferguson, and J. M. Dolan, "Robust mission execution for autonomous urban driving," in 10th International Conference on Intelligent Autonomous Systems (IAS 2008), 2008, pp. 155-163.

[10] B. Pitzer, M. Styer, C. Bersch, C. DuHadway, and J. Becker, "Towards perceptual shared autonomy for robotic mobile manipulation," in IEEE International Conference on Robotics and Automation (ICRA), 2011.

[11] E. Marder-Eppstein, E. Berger, T. Foote, B. P. Gerkey, and K. Konolige, "The office marathon: Robust navigation in an indoor office environment," in International Conference on Robotics and Automation, 05/2010 2010.

[12] N. Dantam and M. Stilman, "The motion grammar: Linguistic perception, planning, and control," in Proceedings of Robotics: Science and Systems, Los Angeles, CA, USA, June 2011.

[13] D. Harel, "Statecharts: A visual formalism for complex systems," Sci. Comput. Program., vol. 8, pp. 231-274, June 1987.

[14] C. Rother, V. Kolmogorov, and A. Blake, "Grabcut: Interactive foreground extraction using iterated graph cuts," ACM Transactions on Graphics, vol. 23, pp. 309-314, 2004.

[15] M. Quigley, K. Conley, B. P. Gerkey, J. Faust, T. Foote, J. Leibs, R. Wheeler, and A. Y. Ng, "Ros: an open-source robot operating system," in ICRA Workshop on Open Source Software, 2009.

[16] W. Garage, "The pr2 robot," http://www.willowgarage.com/pages/pr2.

[17] N. Nilsson, A. I. C. S. International), and S. R. I. M. P. CALIF., A Hierarchical Robot Planning and Execution System, ser. Technical note. Defense Technical Information Center, 1973.

[18] B. Lee and E. A. Lee, "Hierarchical concurrent finite state machines in ptolemy," in Proceedings of the 1998 International Conference on Application of Concurrency to System Design, ser. CSD '98. Washington, DC, USA: IEEE Computer Society, 1998, pp. 34-.

[19] P. J. Lucas, "An object-oriented language system for implementing concurrent, hierarchical, finite state machines," Champaign, IL, USA, Tech. Rep., 1994.

[20] L. P. Kaelbling and T. Lozano-Pérez, "Hierarchical task and motion planning in the now," in ICRA, 2011, pp. 1470-1477. 